\title{
Uplink NOMA in Body Area Networks With Simple Node Pairing Strategies
}

This paper was downloaded from TechRxiv (https://www.techrxiv.org).

\section{LICENSE}

CC BY 4.0

SUBMISSION DATE / POSTED DATE

$22-04-2020$ / 22-04-2020

CITATION

Michaelides, Costas; Pavlidou, Foteini-Niovi (2020): Uplink NOMA in Body Area Networks With Simple Node Pairing Strategies. TechRxiv. Preprint. https://doi.org/10.36227/techrxiv.12152793.v1

$\mathrm{DOI}$

10.36227/techrxiv.12152793.v1 


\title{
Uplink NOMA in Body Area Networks With Simple Node Pairing Strategies
}

\author{
Costas Michaelides, Student Member, IEEE and Foteini-Niovi Pavlidou, Senior Member, IEEE
}

\begin{abstract}
In body area networks (BANs), a node placed near the hub has a significant signal strength advantage. This issue, known as near-far problem, indicates that a near node may serve as a relay for a far node. Currently, the established orthogonal multiple access (OMA) requires the allocation of a specific resource block to a single node. In contrast, the emerging non-orthogonal multiple access (NOMA) allows simultaneous transmissions and uses successive interference cancellation (SIC) to recover the signals. For the first time in the literature, we introduce NOMA in BANs, to allow simultaneous transmissions from two nodes at a time, with distinguishable power levels. The crucial part of this technique is the dynamic pairing of the nodes by the hub. First, we review the fundamentals of uplink NOMA and we propose an access protocol in compliance with IEEE 802.15.6-2012, which combines random OMA with scheduled NOMA. Next, we propose three node pairing strategies, namely a random, a correlative and a conditional one, to meet the requirements of several applications. These strategies are evaluated with packet level simulations in OMNeT++. Our results show that the proposed scheme outperforms the basic OMA scheme at high packet rates and provides a feasible direction for novel relay-based applications.
\end{abstract}

Index Terms-access protocols, body area networks, interference cancellation, non-orthogonal multiple access.

\section{INTRODUCTION}

$\mathbf{N}$ ON-ORTHOGONAL multiple access (NOMA) has received significant attention lately, specifically in the context of 5G (see [1], [2]). The underlying technology, successive interference cancellation (SIC), is a well known capacity achieving technique (see [3]) which has not been exploited yet, due to hardware limitations. In this work, we investigate the feasibility and the potential benefits of powerdomain NOMA in body area networks (BANs) [4], particularly when the sensor nodes are placed on body surface. This is a much requested use case which applies to several medical and entertainment applications. We are particularly interested in the uplink, since the sensor nodes transmit their data packets to a hub, usually a smartphone [5], or a wristband.

NOMA, based on superposition coding and SIC (see [6]), emerged from NTT Docomo as a candidate technology for future radio access [7]. An overview of the contributions from NTT Docomo is provided in [8]. Moreover, in [9] the authors propose an uplink scheme by considering fairness

The authors are with the School of Electrical and Computer Engineering, Aristotle University of Thessaloniki, 54124 Thessaloniki, Greece (e-mail: cmich@auth.gr; niovi@auth.gr). among the users. Also in [10], a power allocation scheme is proposed for the downlink and the uplink. Considering medium access control (MAC), a multichannel ALOHA is proposed in [11]. However, in our work, we focus on node pairing and scheduled access. A valuable work on SIC-aware scheduling is [12]. The proposed method uses power control to maximize the throughput. Additionally, the authors from NTT Docomo elaborate on low complexity access schemes for practical implementations in [13] and [14]. Moreover, in [15], the authors propose dynamic clustering algorithms for the downlink and the uplink to maximize the overall system throughput. Also, in [16] the authors propose user pairing algorithms using channel quality indicator (CQI). In [17], the authors follow the stochastic geometry approach to evaluate a selective user pairing scheme. Lastly, in [18], user pairing is investigated from a combinatorial perspective.

Recently, a few testbeds have been presented, including a software defined radio (SDR) implementation in [19] and a low cost Wi-Fi implementation in [20]. The most remarkable development is credited to NTT Docomo and MediaTek, which performed experimental trials using handheld devices, presented in [21]. These trials indicate that it is the right time to apply NOMA in BANs, since our only prerequisite is a module with SIC capability, to use as a hub.

In this work, we attempt to satisfy the requirements of BANs, particularly low latency, using low complexity scheduling. To the best of our knowledge, this is the first study of NOMA in BANs. Thus, our work is also oriented towards the understanding of its implications. Power-domain NOMA, based on multiplexed signals with distinguishable power levels, motivates us to use a node with a strong signal as a relay for a node with a weak signal using superposition coding. Our purpose is to pair the nodes without any power or rate control. Specifically, we exploit the near-far problem, which is common in BANs due to the attachment of the nodes on the human body. While the pairing of two nodes might be considered as a limitation in other networks, the dynamic pairing of just two nodes in each superframe unlocks a new field of relay-based applications in BANs. In this context, pairing can be treated as a new layer of abstraction. In order to satisfy the requirements of several applications, we propose three pairing strategies for two nodes, which are evaluated with realistic packet level simulations, implemented with Castalia [22], in OMNeT++ [23].

The rest of this article is structured as follows. In Section II, we present the system model. In Section III we elaborate on the proposed node pairing strategies. In Section IV we discuss the simulation results. Section V concludes the article. 


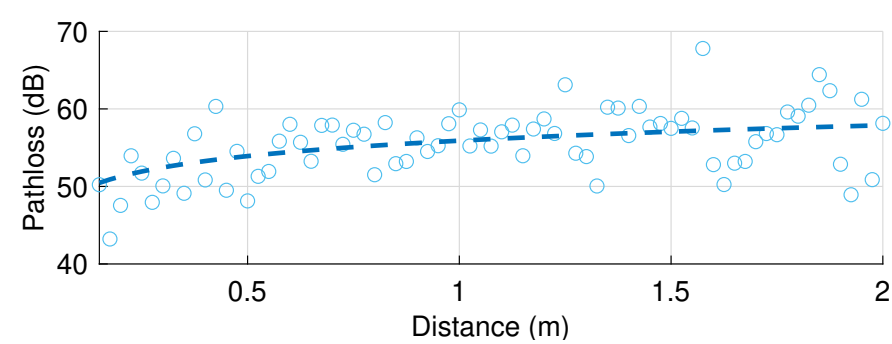

Fig. 1. A random instance of pathloss in $2.4 \mathrm{GHz}$ (CM3A [26]).

\section{SYSTEM MODEL}

In this section we introduce our system model. Our concern is to capture the basic features of BANs, in order to investigate the feasibility of NOMA in realistic conditions. In the following, we elaborate on the most important topics, including mobility, the wireless channel, the physical layer and the data link layer.

\section{A. Mobility}

Mobility is a crucial aspect of BANs. The transmission power levels are low and human body parts are constantly in motion. Thus, a realistic representation of mobility is required, in order to capture any connectivity issues. Here, we use a trace-based mobility model with motion capture data from HDM05 database [24], where a human subject walks inside a small room, grabbing and depositing objects on shelves. We place 1 hub and 9 sensor nodes on the human body. This model describes a situation with moderate mobility in a typical environment which can be associated with several channel models. An animated version of this model can be watched in [25], or the accompanying video of this article.

\section{B. Wireless channel}

According to the measurements from [27], time coherence for walking is $125 \mathrm{~ms}$. In our case, the symbol duration for $512 \mathrm{kbps}$ and 1 bit per symbol is much shorter. Thus, we may assume that the channel does not change during a symbol's transmission. However, we have to take into account pathloss due to the absorption of the human body, which is a major cause of signal degradation. Specifically, we use the lognormal pathloss model CM3A, from the IEEE technical report on the channel model for BANs [26]:

$$
\operatorname{PL}(d)_{\mathrm{dB}}=\alpha \log _{10}(d)+\beta+X\left(\mu, \sigma^{2}\right) .
$$

The distance $d$ is in $\mathrm{mm}$ and the coefficients of linear fitting are: $\alpha=6.6$ and $\beta=36.1 . X$ is a normally distributed variable with $\mu=0$ and $\sigma=3.8 \mathrm{~dB}$. These values are based on measurements in a hospital room, in $2.4 \mathrm{GHz}$. The expected results up to $2 \mathrm{~m}$ are depicted in Fig. 1 . This model, combined with trace-based mobility creates a sufficiently realistic environment with huge pathloss.

\section{Physical layer}

Power-domain NOMA is based on superposition coding (see [28]). Our goal is the detection of symbols from multiple

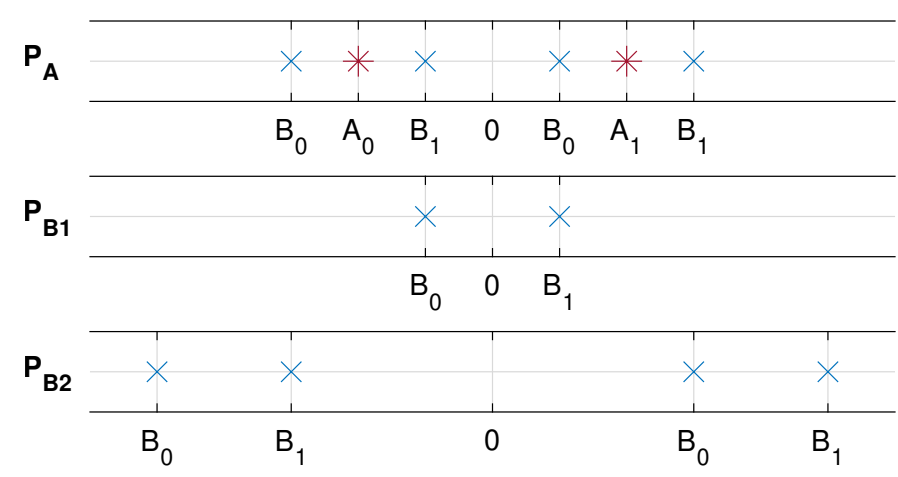

Fig. 2. From top to bottom: two superposed constellations, correct detection of $A_{0}$ or $A_{1}$, wrong detection of $A_{0}$ or $A_{1}$ with error propagation either to the left or to the right.

transmitters on a superposed constellation. Considering the case of two simultaneous signal transmissions with distinct power levels $S_{A}, S_{B}$ and noise density $N_{0}$, the respective rates should satisfy the single user constraints (see [6])

$$
R_{A}<\log _{2}\left(1+\frac{S_{A}}{N_{0}}\right), \quad R_{B}<\log _{2}\left(1+\frac{S_{B}}{N_{0}}\right)
$$

and yet another constraint due to superposition,

$$
R_{A}+R_{B}<\log _{2}\left(1+\frac{S_{A}+S_{B}}{N_{0}}\right) .
$$

This upper bound breaks into

$$
\begin{aligned}
\log _{2}\left(1+\frac{S_{A}+S_{B}}{N_{0}}\right)= & \underbrace{\log _{2}\left(1+\frac{S_{A}}{N_{0}+S_{B}}\right)}_{\text {stage 1 }} \\
& +\underbrace{\log _{2}\left(1+\frac{S_{B}}{N_{0}}\right)}_{\text {stage 2 }}
\end{aligned}
$$

which unveils that a non zero rate for each node is feasible, provided that the detection takes place in two stages. In stage 1 , the receiver attempts to detect $S_{A}$, while $S_{B}$ is treated as Gaussian interference. In stage 2, after a successful detection and subtraction of the signal, $S_{B}$ is free of interference.

The aforementioned method, known as SIC, assumes an effective detection strategy at the receiver. In order to model the uplink of BANs, we are interested in the superposition of two BPSK or two QPSK constellations. Here, we use BPSK. The results for QPSK are similar (see [29], [30]). In the case of two nodes, a received signal by the hub is:

$$
r=A_{i}+B_{j}+n, \quad i, j \in[0,1],
$$

where $A_{i}, B_{j}$, are the symbols from node $\mathrm{A}$ and node $\mathrm{B}$ respectively and $n$ is the additive white Gaussian noise (AWGN), with mean value $\mu=0$ and variance $\sigma^{2}=N_{0} / 2$. Please note that the transmission powers are equal and the channel gains are included in $A_{i}, B_{j}$. Equation (5) implies that we have two superposed constellations in signal space, as depicted in Fig. 2. Note that the minimum distances between the symbols of each constellation are $2 d_{A}$ and $2 d_{B}$ respectively, where $d_{A}=\sqrt{\left(\mathcal{E}_{b}\right)_{A}}$ and $d_{B}=\sqrt{\left(\mathcal{E}_{b}\right)_{B}}$ in terms of energy. 
Additionally, we assume that node A has a stronger signal than node $\mathrm{B}$.

In Fig. 2, upon a signal reception, the hub attempts to detect the symbol of the strong signal $\left(A_{i}\right)$. Subsequently, it subtracts the detected symbol and attempts to detect the symbol of the weak signal $\left(B_{j}\right)$. If $A_{i}$ is detected successfully, there is a strong possibility that the rest of the signal will suffice for a successful detection of $B_{j}$. However, a wrong detection of $A_{i}$ causes error propagation either to the left or to the right.

The operations in Fig. 2 can be described with the following probabilities. From top to bottom, $P_{A}$ is the probability of wrong detection of $A_{i}, P_{B 1}$ is the probability of wrong detection of $B_{j}$ provided that the detection of $A_{i}$ is correct and $P_{B 2}$ is the probability of wrong detection of $B_{j}$ provided that the detection of $A_{i}$ is wrong. See [29], [30] for the detailed derivations. The bit error probabilities $P_{A}$ and $P_{B}=P_{B 1}+P_{B 2}$ are expressed in terms of the $Q$-function:

$$
\begin{aligned}
P_{A}= & \frac{1}{2}\left[Q\left(\frac{d_{A}+d_{B}}{\sqrt{N_{0} / 2}}\right)+Q\left(\frac{d_{A}-d_{B}}{\sqrt{N_{0} / 2}}\right)\right], \\
P_{B}= & \frac{1}{2}\left[2 Q\left(\frac{d_{B}}{\sqrt{N_{0} / 2}}\right)-Q\left(\frac{d_{A}+d_{B}}{\sqrt{N_{0} / 2}}\right)\right. \\
+ & Q\left(\frac{2 d_{A}+d_{B}}{\sqrt{N_{0} / 2}}\right)-Q\left(\frac{2 d_{A}-d_{B}}{\sqrt{N_{0} / 2}}\right) \\
& \left.+Q\left(\frac{d_{A}-d_{B}}{\sqrt{N_{0} / 2}}\right)\right] .
\end{aligned}
$$

Note that the $Q$-function, defined as

$$
Q\left(\frac{x-\mu}{\sigma}\right)=\frac{1}{\sqrt{2 \pi}} \int_{\frac{x-\mu}{\sigma}}^{\infty} \mathrm{e}^{-\frac{y^{2}}{2}} \mathrm{~d} y=P\left(y>\frac{x-\mu}{\sigma}\right)
$$

gives the tail probability of normal distribution. We substitute the $Q$-function with the complimentary error function,

$$
Q(x)=\frac{1}{2} \operatorname{erfc}\left(\frac{x}{\sqrt{2}}\right),
$$

which eliminates $\sqrt{2}$ and assists our calculations using $\mathrm{C}++$ math library. Also, we substitute the $\mathcal{E}_{b} / N_{0}$ of node $\mathrm{A}$ and node B with $\gamma_{\alpha}$ and $\gamma_{\beta}$ respectively to simplify the notation. Thus, the bit error probabilities $P_{A}$ and $P_{B}$ are transformed into the following convenient expressions:

$$
\begin{aligned}
P_{A}= & \frac{1}{4}\left[\operatorname{erfc}\left(\sqrt{\gamma_{\alpha}}+\sqrt{\gamma_{\beta}}\right)+\operatorname{erfc}\left(\sqrt{\gamma_{\alpha}}-\sqrt{\gamma_{\beta}}\right)\right], \\
P_{B}= & \frac{1}{4}\left[2 \operatorname{erfc}\left(\sqrt{\gamma_{\beta}}\right)-\operatorname{erfc}\left(\sqrt{\gamma_{\alpha}}+\sqrt{\gamma_{\beta}}\right)\right. \\
& +\operatorname{erfc}\left(2 \sqrt{\gamma_{\alpha}}+\sqrt{\gamma_{\beta}}\right)-\operatorname{erfc}\left(2 \sqrt{\gamma_{\alpha}}-\sqrt{\gamma_{\beta}}\right) \\
& \left.+\operatorname{erfc}\left(\sqrt{\gamma_{\alpha}}-\sqrt{\gamma_{\beta}}\right)\right] .
\end{aligned}
$$

Fig. 3, includes these bit error probabilities (BEP) versus signal to noise ratio (SNR), where $\gamma_{\alpha_{\mathrm{dB}}}$ is constant and $\gamma_{\beta_{\mathrm{dB}}}$ is variable. The BEP of basic BPSK from [6],

$$
P_{\mathrm{BPSK}}=Q\left(\sqrt{\frac{2 \mathcal{E}_{b}}{N_{0}}}\right)=\frac{1}{2} \operatorname{erfc}\left(\sqrt{\gamma_{\beta}}\right)
$$

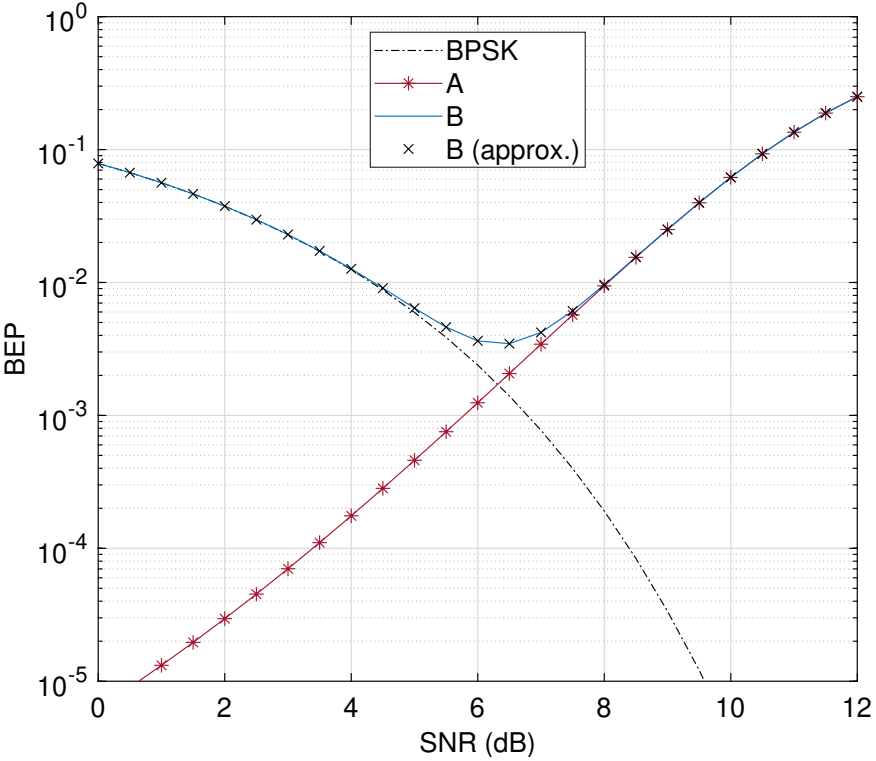

Fig. 3. BEP curves: $\gamma_{\alpha_{\mathrm{dB}}}=12 \mathrm{~dB}$ and $\gamma_{\beta_{\mathrm{dB}}}$ is variable, up to $12 \mathrm{~dB}$.

is also included as a reference. We notice that $P_{B}$ follows $P_{\text {BPSK }}$, it gets a minimum value and subsequently it converges with $P_{A}$, as $\gamma_{\beta_{\mathrm{dB}}}$ approaches $\gamma_{\alpha_{\mathrm{dB}}}$. In order to calculate this local minimum we use an approximation of $P_{B}$, depicted in Fig. 3. Specifically, we select the first and the last element of $P_{B}$, which match well the convex area. Thus, the approximate expression is

$$
P_{B} \approx \frac{1}{4}\left(2 \operatorname{erfc}\left(\sqrt{\gamma_{\beta}}\right)+\operatorname{erfc}\left(\sqrt{\gamma_{\alpha}}-\sqrt{\gamma_{\beta}}\right)\right) .
$$

By differentiating with respect to $\gamma_{\beta}$ we get

$$
\frac{d P_{B}}{d \gamma_{\beta}}=\frac{1}{2 \sqrt{\pi \gamma_{\beta}}}\left(e^{-\left(\sqrt{\gamma_{\alpha}}-\sqrt{\gamma_{\beta}}\right)^{2}}-e^{-\gamma_{\beta}}\right) .
$$

Finally, for $d P_{B} / d \gamma_{\beta}=0$ we get a handy rule of thumb:

$$
\gamma_{\beta}=\frac{\gamma_{\alpha}}{4}
$$

which can be approximated in $\mathrm{dB}$ with

$$
\gamma_{\beta_{\mathrm{dB}}}=10 \log _{10}\left(\frac{\gamma_{\alpha}}{4}\right) \approx \gamma_{\alpha_{\mathrm{dB}}}-6 \mathrm{~dB}
$$

This is mainly an indication rather than a strict constraint, since we are dealing with probabilities. However, as an indication of the SIC capability of the receiver, it could be useful during the pairing of nodes with low SNRs.

In practice, interference is the predominant limitation in BANs due to short distances. Thus, we use signal to interference plus noise ratio (SINR) instead of SNR. In this work, we use the additive interference model of Castalia, which may be simplistic but it captures well the effect of interference. For each received signal, every other signal is considered as interference. The total power of interference is subtracted from the power of the received signal before the detection. In order to perform SIC, we enhanced the probabilistic detection model of Castalia with $P_{A}$ and $P_{B}$, as expressed by the Equations (10) and (11) respectively. 

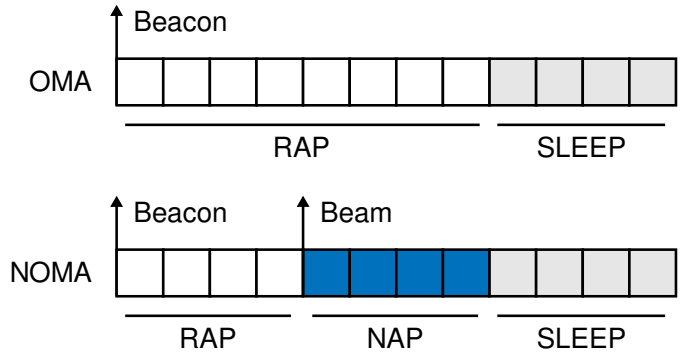

Fig. 4. Superframes for OMA and NOMA, repeated over time.

\begin{tabular}{|c|c|c|c|}
\hline \multicolumn{1}{c}{7 bytes } & \multicolumn{1}{c}{6 bytes } & \multicolumn{1}{c}{6 bytes } & 1 byte \\
\hline Header & $\begin{array}{r}\text { Recipient } \\
\text { Address A }\end{array}$ & $\begin{array}{r}\text { Recipient } \\
\text { Address B }\end{array}$ & $\begin{array}{c}\text { NAP } \\
\text { Length }\end{array}$ \\
\hline
\end{tabular}

Fig. 5. Beam frame.

\section{Data link layer}

The basic superframe of IEEE 802.15.6-2012 for orthogonal multiple access (OMA) includes a random access phase (RAP). In order to support NOMA, we added a NOMA phase (NAP) after RAP, as depicted in Fig. 4. At the beginning of each superframe, the hub transmits a beacon to inform the connected nodes about the upcoming RAP. During RAP, the nodes transmit their data packets using carrier-sense multiple access with collision avoidance (CSMA/CA). The hub stores the received signal strength indication (RSSI) value, included in the payload of each packet. At the end of each RAP, the hub calculates the average RSSI value of each node and performs an RSSI-based node pairing strategy to select a node with strong signal (as node A) and a node with weak signal (as node B); more on this in Section III. Subsequently, the hub transmits a beam, depicted in Fig. 5, to inform the paired nodes about the upcoming NAP. At this point, the hub switches to SIC mode and the paired nodes may transmit their data packets simultaneously, until the end of NAP. The aforementioned procedure is depicted in Fig. 6.

A special note is required on the delay due to SIC. In [31], a software implementation in GNU Radio [32], the delay is about a few ms. This is not a major issue concerning latency, due to the expected benefits of simultaneous transmissions. However, this delay affects the timing of the protocol, specifically the reception of Ack packets by the nodes after a successful detection. In this work, we do not take into account SIC delay due to the lack of any specific values. Our approach, is to use an extended TX interval, including two Acks and their interframe spaces,

$$
T_{\text {packet }}+2 \times T_{\text {ack }}+4 \times(\text { pMIFS }+ \text { pExtraIFS }),
$$

to allow enough time for both SIC and Ack reception.

\section{NODE PAIRING STRATEGIES}

The nodes in BANs suffer from huge pathloss due to the absorption of human body. Thus, we may exploit the nearfar problem, in order to pair two nodes with distinguishable signal strength values without any power or rate control.

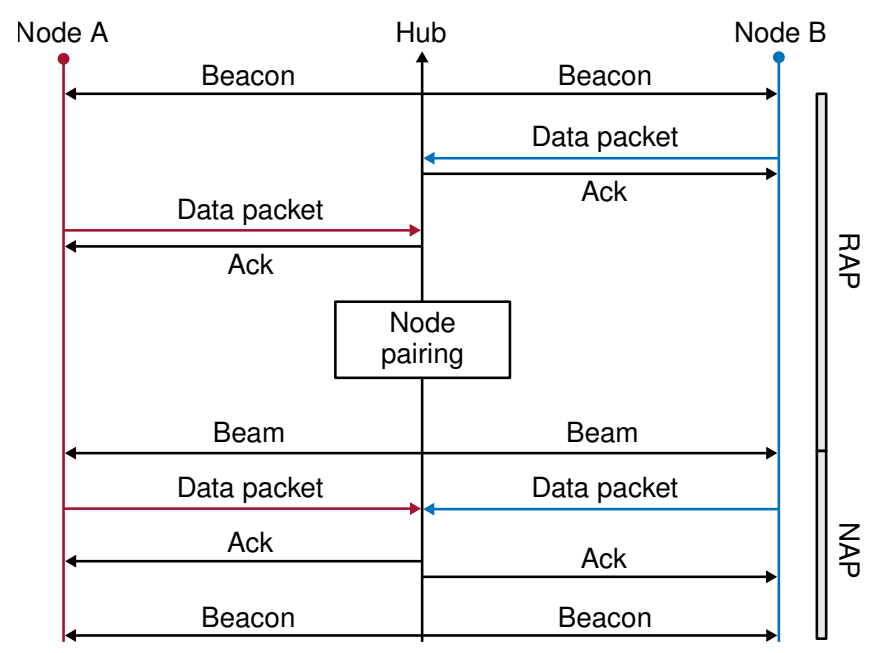

Fig. 6. Communication during a superframe.

Upon the selection of node A, the signal strength of node B should preferably satisfy (15), in order to minimize the error probability. However, Fig. 3 shows that for high SNRs, $P_{B}$ is expected to drop at very low values. Thus, provided that we use BPSK signals, this constraint can be relaxed.

The proposed pairing strategies exploit pathloss, using the RSSI values of the packets, on par with our previous work [33]. The hub keeps the statistics of the received packets and pairs two nodes in each superframe, according to its current strategy. The very basic strategy is random pairing, which seeks fairness among the nodes, from the perspective of data link layer. Correlative pairing attempts to satisfy (15), which correlates the signal strengths of two nodes. Lastly, conditional pairing satisfies network and application specific requirements. In the following, we elaborate on these strategies.

\section{A. Random pairing}

The hub stores the RSSI values from the received packets and calculates an average SNR value for each node and a total average SNR. The nodes with SNR above the total average are put into group A and the rest of the nodes are put into group B. Subsequently, node A is picked randomly from group A, while node B is picked randomly from group B. Random pairing uses a relaxed pairing constraint and promotes fairness in an effortless way. Thus, it may serve as a fallback when any other strategy is not feasible.

\section{B. Correlative pairing}

Correlative pairing takes into account the SIC capability of the hub. First, the nodes are put into groups $\mathrm{A}$ and B, according to their estimated SNRs. Node A is picked randomly from group A. However, the selection of node B is correlated to the SNR value of the selected node A. In our case, the superposition of two BPSK signals, we select the best matching node from group B, according to (15). This procedure, depicted in Fig. 7, is ideal when the nodes have low SNRs, provided that they have packets to sent. 


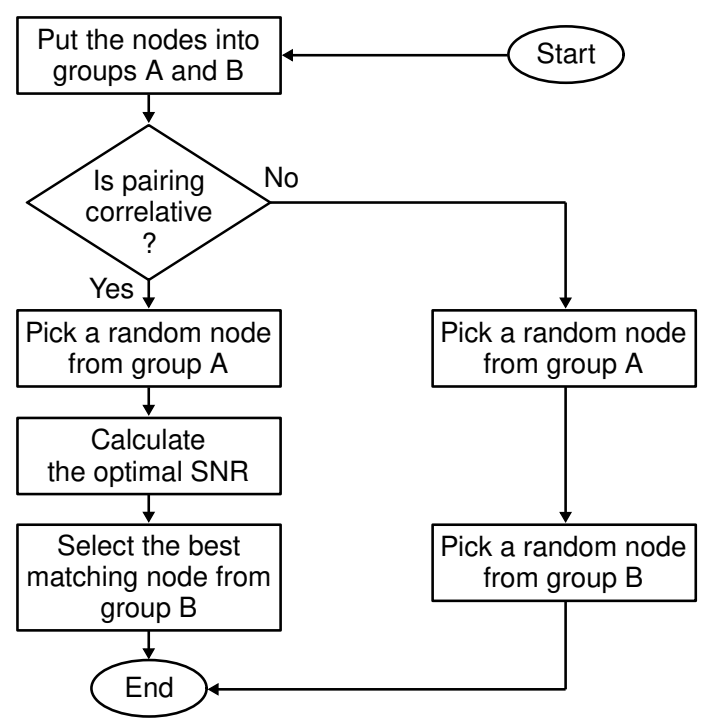

Fig. 7. Correlative node pairing.

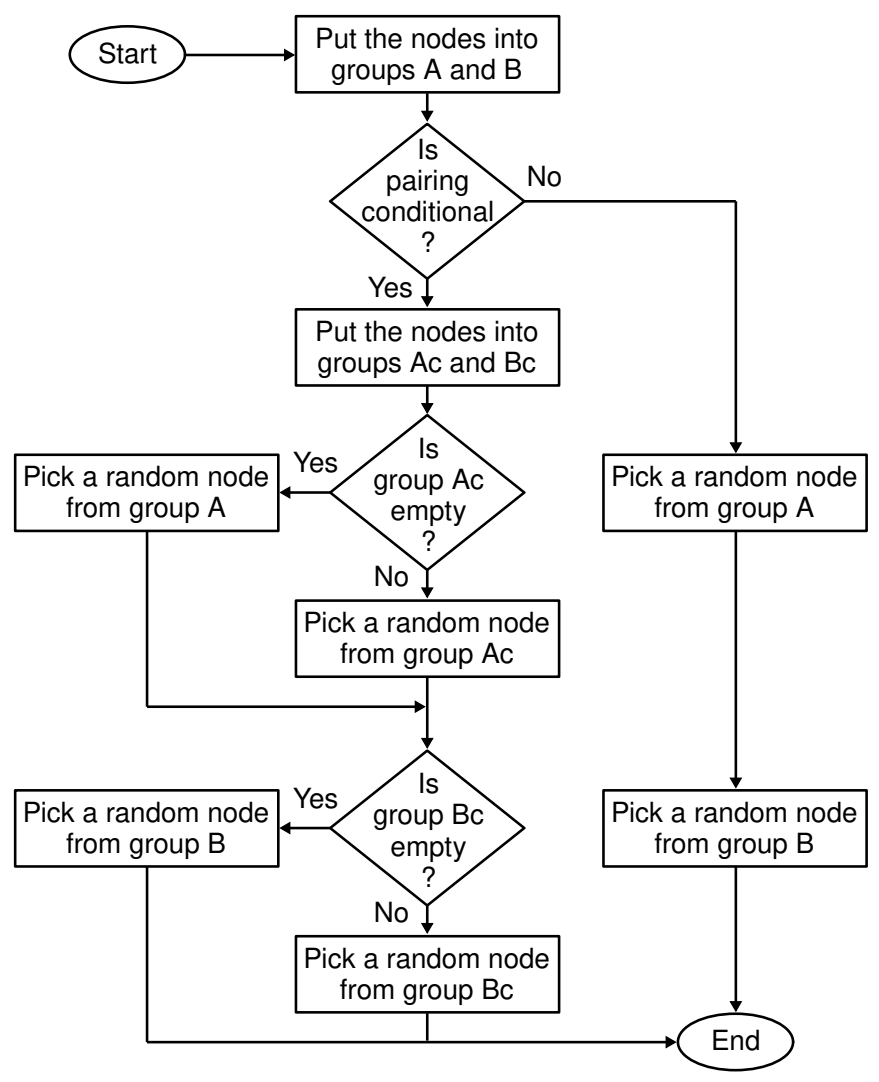

Fig. 8. Conditional node pairing.

\section{Conditional pairing}

Conditional pairing introduces an optional constraint which can be set from upper layers. Again, the nodes are put into groups A and B. Next, the nodes which satisfy a condition are put into $\mathrm{Ac}$ and $\mathrm{Bc}$ respectively. Thus, node $\mathrm{A}$ and node $\mathrm{B}$ are picked randomly from $\mathrm{Ac}$ and $\mathrm{Bc}$. The complete flow of events is included in Fig. 8. This strategy blends filtering with randomness and provides several opportunities for pairing.
TABLE I

SIMULATION PARAMETERS

\begin{tabular}{|c|c|c|}
\hline & Parameter & Value \\
\hline \multirow[t]{7}{*}{ Application } & Time & $60 \mathrm{~s}$ \\
\hline & Area & $4 \mathrm{~m} \times 2 \mathrm{~m} \times 2 \mathrm{~m}$ \\
\hline & Hub & 1 \\
\hline & Nodes & 9 \\
\hline & Packet rate & $20-100$ packets/s \\
\hline & Data payload & 100 bytes \\
\hline & Header overhead & 5 bytes \\
\hline Network & NET packet overhead & 10 bytes \\
\hline \multirow[t]{6}{*}{ MAC } & Slot duration & $10 \mathrm{~ms}$ \\
\hline & Superframe & 12 slots \\
\hline & Access method (RAP) & Slotted CSMA/CA [4] \\
\hline & Access method (NAP) & NOMA \\
\hline & Buffer & 16 packets \\
\hline & MAC frame overhead & 7 bytes \\
\hline \multirow[t]{11}{*}{ PHY } & Carrier frequency & $2.4 \mathrm{GHz}$ \\
\hline & Modulation & BPSK \\
\hline & Bits per symbol & 1 \\
\hline & Bit rate & $512 \mathrm{kbps}$ \\
\hline & Noise bandwidth & $100 \mathrm{kHz}$ \\
\hline & Noise floor & $-104 \mathrm{dBm}$ \\
\hline & Sensitivity & $-90 \mathrm{dBm}$ \\
\hline & $\mathrm{RX}$ power & $3.1 \mathrm{~mW}$ \\
\hline & TX power & $3.0 \mathrm{~mW}(-10 \mathrm{dBm})$ \\
\hline & Sleep power & $0.05 \mathrm{~mW}$ \\
\hline & PHY frame overhead & 6 bytes \\
\hline Channel & Pathloss model & Lognormal: CM3A [26] \\
\hline Mobility & Trace based model & $\begin{array}{l}\text { Grabbing and depositing } \\
\text { objects in a room [25] }\end{array}$ \\
\hline
\end{tabular}

Two useful use cases are: a buffer-aware pairing and a priorityaware pairing. A buffer-aware pairing puts a node into Ac or $\mathrm{Bc}$, if its buffered packets are more than the average buffered packets. A priority-aware pairing puts a node into $\mathrm{Ac}$ or $\mathrm{Bc}$, if its packets are marked as high-priority.

\section{Simulation Results}

In this section, we evaluate the proposed pairing strategies, specifically random, correlative and conditional, using 1 hub and 9 sensor nodes in star topology, placed on the human body (see Fig. 9). The simulations are implemented with Castalia, in OMNeT++. Our metrics are the received beams, the sent data packets, the received data packets and application level latency. The provided results, received after 100 realizations of each case, are compared to the results of a baseline OMA scheme, in compliance with the standard.

The most important parameters are included in Table I. Please note that the performance of the proposed strategies depends heavily on the duration of the access phases. Here, the baseline OMA scheme uses a RAP with 8 slots, while the proposed scheme uses a RAP with 4 slots and a NAP with 4 slots, as depicted in Fig. 4. This arrangement is not restrictive. In our case, it provides comparable, intuitive results and allows us to identify the pros and cons of each pairing strategy. 


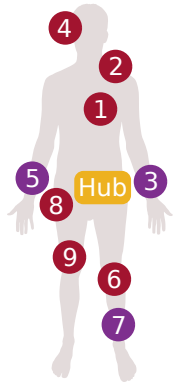

Fig. 9. Node placement.

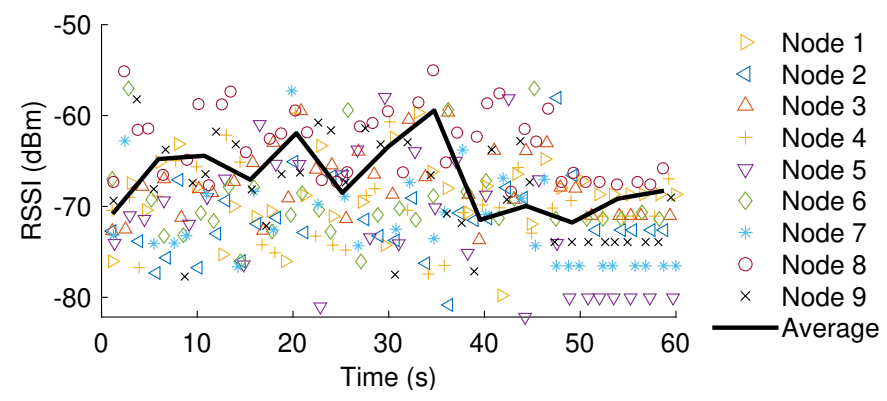

Fig. 10. A few RSSI traces during one realization.

Fig. 10 includes a few RSSI traces, during one realization of the simulation. This figure unveils the feasibility of node pairing without power control. We notice that the RSSI values of the nodes lie in specific intervals. Even if the nodes move, they are still restricted due to their attachment on the human body. Thus, we have several opportunities for pairing. An obvious pitfall is that we may have recurring pairs, due to the aforementioned patterns. Hopefully, the employed randomness during pairing will remedy this issue.

Fig. 11 and Fig. 12 provide an overview of the received packets for a variable packet rate, from 20 packets/s to 100 packets/s. At low packet rates NAP is under-utilized due to the fact that the paired nodes produce very few packets. In Fig. 11, we notice that the baseline is already saturated at 50 packets/s, while the received packets with NOMA increase up to 100 packets/s, due to the simultaneous transmissions during NAP. In Fig. 12, the received packets with latency below 100 ms show that NOMA outperforms OMA at high packet rates.

The rest of the results are specific to 50 packets/s. At this point, the performance of the baseline is saturated. This allows us to focus on the performance of each pairing strategy. Fig. 13 and Fig. 14 show the recipients of the transmitted beams. Fig. 15 and Fig. 16 show the sent and received packets respectively. Fig. 17 shows the reasons for packet failure from the data link layer perspective. Lastly, Fig. 18 shows the received packets with respect to application level latency, i.e. the time interval from the creation of a packet until its delivery.

Random pairing performs very well, with minimal complexity. As described in Section III, the two nodes are picked randomly from two groups. This is indeed a very effective strategy, since the SNRs of the nodes lie in certain intervals, as exhibited in Fig. 10. Random pairing succeeds particularly

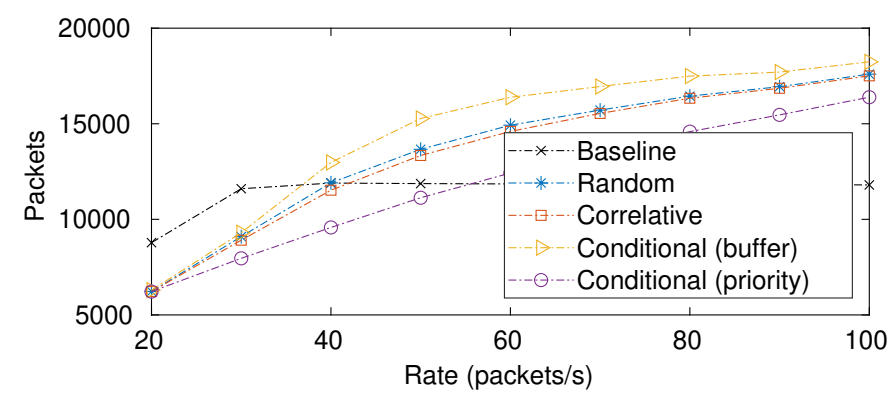

Fig. 11. Received data packets at the hub.

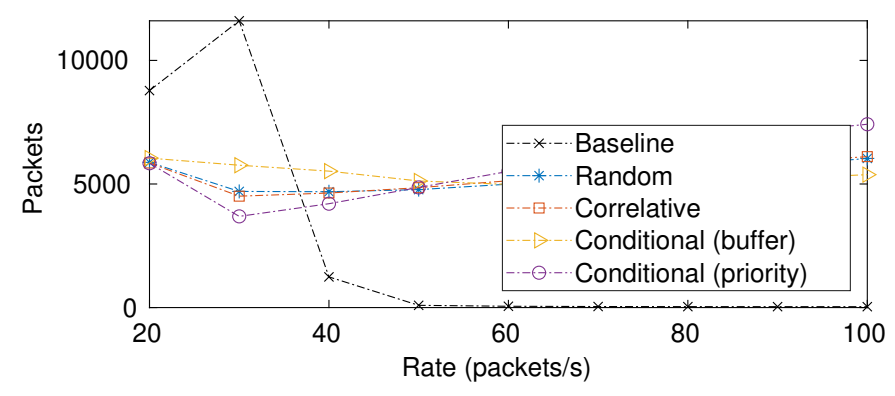

Fig. 12. Received data packets at the hub with latency below $100 \mathrm{~ms}$.

in terms of fairness. In Fig. 13, node 8 dominates as recipient $\mathrm{A}$, due to the fact that it is regularly present in group A. However, in Fig. 14, the selection of recipient B is balanced. In Fig. 15 and in Fig. 16, it seems that random pairing has similar results compared to the baseline. However, Fig. 18, unveils that random pairing has much lower latency. Additionally, Fig. 17 indicates a reduced, but still significant number of missed Acks, due to pathloss or failed SIC.

Correlative pairing exploits the SIC capability and attempts an optimal selection of recipient $\mathrm{B}$, according to the SNR of recipient A. The received beams in Fig. 13 and Fig. 14 are quite similar to random pairing. However, in Fig. 15 and Fig. 16, it under-performs in terms of the sent and received data packets. Its weakness is that recipient B might not have any packets to sent, while other nodes may be more in need. This is visible in Fig. 17, where buffer overflow is increased compared to random pairing. However, depending on SIC capability and the channel conditions, this strategy might be more suitable instead of an entirely random selection.

Conditional pairing serves as an abstraction for high layers and has the most interesting operation because it enables programmable pairing. Buffer-aware pairing serves low latency applications. Fig. 11 shows that it outperforms the other pairing strategies in terms of packet reception. This is also depicted in Fig. 17, with reduced buffer overflow. Also, Fig. 18 shows that it is very effective in terms of latency. Priorityaware pairing is an application specific strategy, which favors certain nodes. For example, nodes 3, 5 and 7 may represent Einthoven's triangle, the basic setup for electrocardiography (ECG). Fig. 13 and Fig. 14, show that these nodes are favored which results in high packet reception in Fig. 16. Inevitably, in Fig. 17, priority aware pairing experiences increased buffer overflow due to the rest of the nodes. 


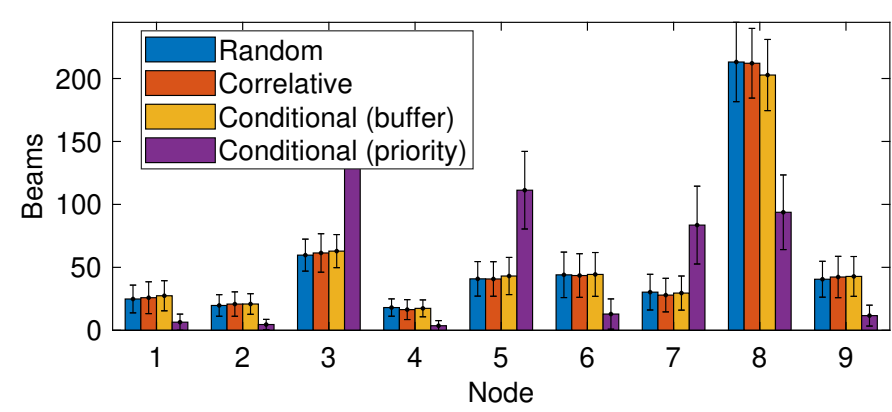

Fig. 13. Received beams at each node (Recipient A, at 50 packets/s).

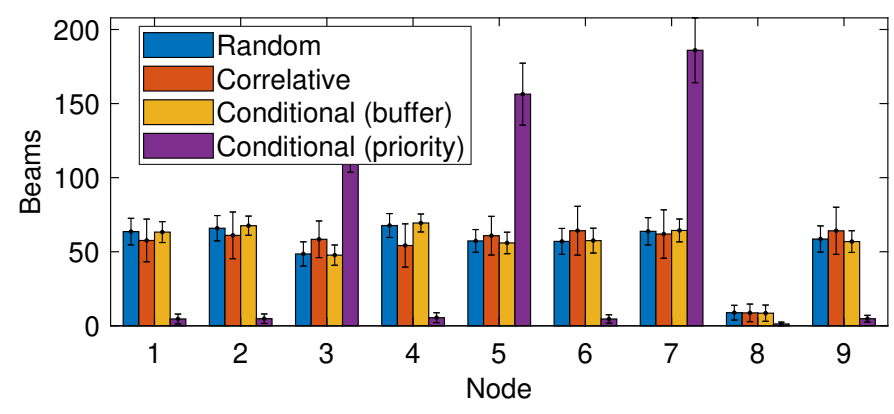

Fig. 14. Received beams at each node (Recipient B, at 50 packets/s).

A general remark concerning NOMA is that it shines at high packet rates, where OMA is saturated. However, the proposed scheme reserves some slots in each superframe for the paired nodes, which means that access is denied to every other node. This may result in unnecessary complicated scheduling at low packet rates, where OMA operates efficiently without any configuration. Thus, the coexistence of OMA and NOMA seems the most reasonable direction. The critical point where NOMA could be enabled is an upper latency threshold, which is essentially a sign of saturation.

Depending on the hardware implementation, the required signal processing for SIC implies increased delay and energy consumption. Thus, NOMA is desirable only in the uplink of BANs, using a capable device as a hub, such as a smartphone. Thanks to central coordination, the sensor nodes may benefit from increased capacity without any overhead.

The dynamic pairing of two nodes indicates an interesting, alternative relaying technique which may satisfy several use cases. Random pairing is an effective strategy which can be used as an out-of-the-box feature. Correlative pairing is useful in cases where random pairing might be inefficient, particularly at low SNRs. Conditional pairing provides an abstraction to the upper layers, which allows the deployment of several applications.

To sum up, we highlight once again the fact that NOMA is particularly beneficial in terms of latency (see Fig. 12 and Fig. 18). The feasibility of uplink NOMA in BANs allows us to reconsider the established relaying methods and to exploit node pairing as an abstraction for novel relay-based schemes, targeted at low latency applications. Thus, the proposed node pairing strategies may serve as a first step for further research and eventually an experimental evaluation.

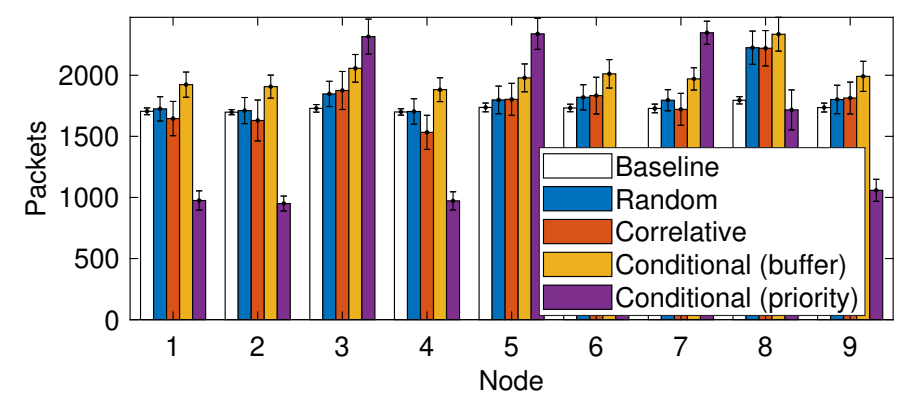

Fig. 15. Sent data packets by each node (at 50 packets/s).

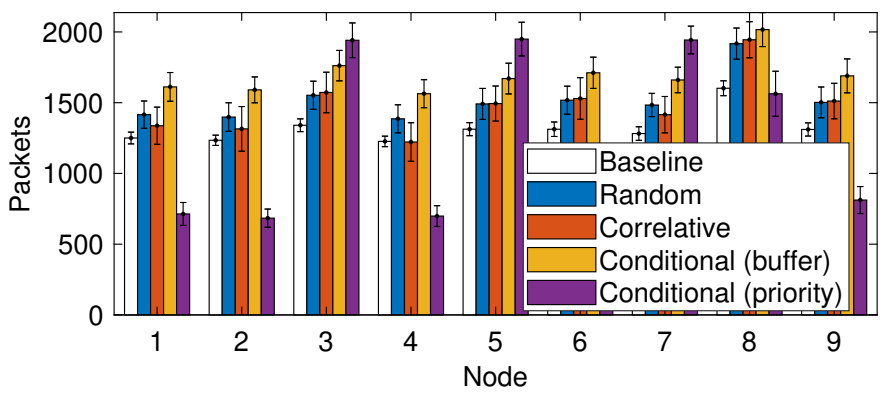

Fig. 16. Received data packets at the hub (at 50 packets/s).

\section{CONCLUSION}

In this article, for the first time in the literature, we presented an implementation of power-domain NOMA in the uplink of BANs, which exploits the near-far problem. Specifically, the near nodes were used as relays of the far nodes using superposition coding, without any power or rate control. For this purpose, we proposed an access protocol and three node pairing strategies from different perspectives, namely random, correlative and conditional, which target the requirements of BANs, particularly low latency. Having in mind that NOMA is expected to serve novel applications rather than legacy ones, we hope that these strategies could inspire further works on several aspects of NOMA in BANs.

\section{REFERENCES}

[1] Z. Ding, X. Lei, G. K. Karagiannidis, R. Schober, J. Yuan, and V. K. Bhargava, "A survey on non-orthogonal multiple access for $5 \mathrm{~g}$ networks: Research challenges and future trends," IEEE Journal on Selected Areas in Communications, vol. 35, no. 10, pp. 2181-2195, Oct 2017.

[2] S. M. R. Islam, N. Avazov, O. A. Dobre, and K. Kwak, "Power-domain non-orthogonal multiple access (noma) in $5 \mathrm{~g}$ systems: Potentials and challenges," IEEE Communications Surveys Tutorials, vol. 19, no. 2, pp. 721-742, Oct 2016

[3] T. Cover, "Broadcast channels," IEEE Transactions on Information Theory, vol. 18, no. 1, pp. 2-14, Jan 1972.

[4] IEEE Standard for Local and metropolitan area networks - Part 15.6: Wireless Body Area Networks, IEEE Std. 802.15.6, Feb. 2012.

[5] G. Fortino, R. Giannantonio, R. Gravina, P. Kuryloski, and R. Jafari, "Enabling effective programming and flexible management of efficient body sensor network applications," IEEE Transactions on HumanMachine Systems, vol. 43, no. 1, pp. 115-133, Jan 2013.

[6] D. Tse and P. Viswanath, Fundamentals of wireless communication. Cambridge university press, 2005.

[7] Y. Saito, Y. Kishiyama, A. Benjebbour, T. Nakamura, A. Li, and K. Higuchi, "Non-orthogonal multiple access (noma) for cellular future radio access," in 2013 IEEE 77th Vehicular Technology Conference (VTC Spring), Jun 2013, pp. 1-5. 


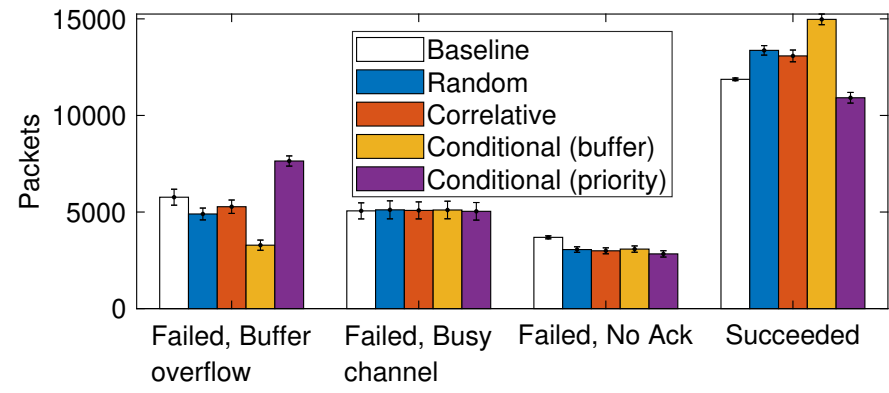

Fig. 17. Data packet breakdown (at 50 packets/s).

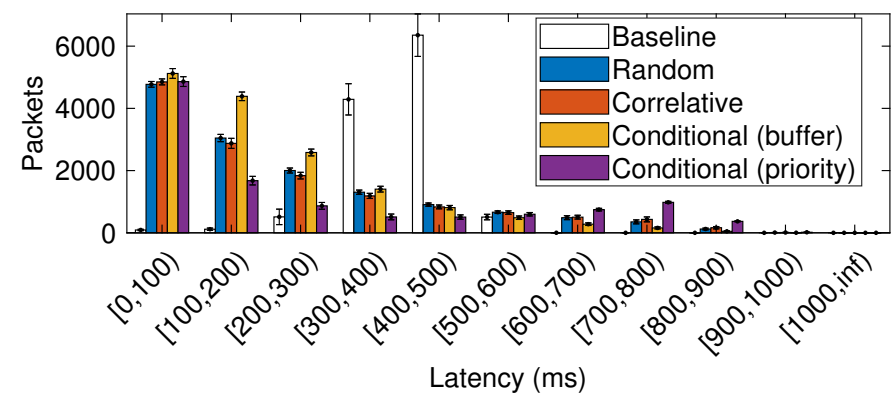

Fig. 18. Application level latency (at 50 packets/s).

[8] K. Higuchi and A. Benjebbour, "Non-orthogonal multiple access (noma) with successive interference cancellation for future radio access," IEICE Transactions on Communications, vol. 98, no. 3, pp. 403-414, Mar 2015.

[9] M. Al-Imari, P. Xiao, M. A. Imran, and R. Tafazolli, "Uplink nonorthogonal multiple access for 5g wireless networks," in 2014 11th International Symposium on Wireless Communications Systems (ISWCS), Aug 2014, pp. 781-785.

[10] Z. Yang, Z. Ding, P. Fan, and N. Al-Dhahir, "A general power allocation scheme to guarantee quality of service in downlink and uplink noma systems," IEEE Transactions on Wireless Communications, vol. 15, no. 11, pp. 7244-7257, Nov 2016.

[11] J. Choi, "Noma-based random access with multichannel aloha," IEEE Journal on Selected Areas in Communications, vol. 35, no. 12, pp. 27362743, Dec 2017.

[12] S. Sen, N. Santhapuri, R. R. Choudhury, and S. Nelakuditi, "Successive interference cancellation: Carving out mac layer opportunities," IEEE Transactions on Mobile Computing, vol. 12, no. 2, pp. 346-357, Feb 2013.

[13] X. Chen, A. Benjebbour, A. Li, and A. Harada, "Multi-user proportional fair scheduling for uplink non-orthogonal multiple access (noma)," in 2014 IEEE 79th Vehicular Technology Conference (VTC Spring), May 2014 , pp. 1-5.

[14] A. Li, A. Benjebbour, X. Chen, H. Jiang, and H. Kayama, "Uplink non-orthogonal multiple access (noma) with single-carrier frequency division multiple access (sc-fdma) for $5 \mathrm{~g}$ systems," IEICE Transactions on Communications, vol. 98, no. 8, pp. 1426-1435, Aug 2015.

[15] M. S. Ali, H. Tabassum, and E. Hossain, "Dynamic user clustering and power allocation for uplink and downlink non-orthogonal multiple access (noma) systems," IEEE Access, vol. 4, pp. 6325-6343, Aug 2016.

[16] F. W. Murti and S. Y. Shin, "User pairing schemes based on channel quality indicator for uplink non-orthogonal multiple access," in 2017 Ninth International Conference on Ubiquitous and Future Networks (ICUFN), Jul 2017, pp. 225-230.

[17] Z. Zhang, H. Sun, and R. Q. Hu, "Downlink and uplink non-orthogonal multiple access in a dense wireless network," IEEE Journal on Selected Areas in Communications, vol. 35, no. 12, pp. 2771-2784, Dec 2017.

[18] M. A. Sedaghat and R. R. Müller, "On user pairing in uplink noma," IEEE Transactions on Wireless Communications, vol. 17, no. 5, pp. 3474-3486, May 2018.

[19] S. Abeywickrama, L. Liu, Y. Chi, and C. Yuen, "Over-the-air implementation of uplink noma," in GLOBECOM 2017 - 2017 IEEE Global Communications Conference, Dec 2017, pp. 1-6.

[20] E. Khorov, A. Kureev, and I. Levitsky, "Noma testbed on wi-fi," in
2018 IEEE 29th Annual International Symposium on Personal, Indoor and Mobile Radio Communications (PIMRC), Sep 2018, pp. 1153-1154.

[21] A. Benjebbour, Y. Kishiyama, Y. Okumura, C. Hwang, and I. Fu, "Outdoor experimental trials of advanced downlink noma using smartphonesized devices," in 2018 IEEE 87th Vehicular Technology Conference (VTC Spring), Jun 2018, pp. 1-6.

[22] Castalia. [Online]. Available: https://github.com/boulis/Castalia/

[23] OMNeT++. [Online]. Available: https://www.omnetpp.org/

[24] M. Müller, T. Röder, M. Clausen, B. Eberhardt, B. Krüger, and A. Weber, "Documentation mocap database hdm05," Universität Bonn, Tech. Rep. CG-2007-2, Jun 2007.

[25] Trace based mobility for body area networks: Grabbing and depositing objects. [Online]. Available: https://youtu.be/AV1dNt596Fs

[26] K. Y. Yazdandoost and K. Sayrafian-Pour, "Channel model for body area network (ban)," IEEE, Tech. Rep. 15-08-0780-11-0006, Nov 2010.

[27] R. Fu, Y. Ye, and K. Pahlavan, "Characteristic and modeling of human body motions for body area network applications," International Journal of Wireless Information Networks, vol. 19, no. 3, pp. 219-228, Sep 2012.

[28] T. M. Cover and J. A. Thomas, Elements of information theory. John Wiley \& Sons, Inc., 2006.

[29] X. Wang, F. Labeau, and L. Mei, "Closed-form ber expressions of qpsk constellation for uplink non-orthogonal multiple access," IEEE Communications Letters, vol. 21, no. 10, pp. 2242-2245, Oct 2017.

[30] X. Liu, Z. Chen, Y. Wang, F. Zhou, Y. Luo, and R. Q. Hu, "Ber analysis of noma-enabled visible light communication systems with different modulations," IEEE Transactions on Vehicular Technology, pp. 1-1, Sep 2019.

[31] S. Vanka, S. Srinivasa, Z. Gong, P. Vizi, K. Stamatiou, and M. Haenggi, "Superposition coding strategies: Design and experimental evaluation," IEEE Transactions on Wireless Communications, vol. 11, no. 7, pp. 2628-2639, Jul 2012.

[32] GNU Radio. [Online]. Available: https://www.gnuradio.org/

[33] C. Michaelides and F. Pavlidou, "Programmable mac in body area networks, one command at a time," IEEE Sensors Letters, vol. 3, no. 7, pp. 1-4, Jul 2019.

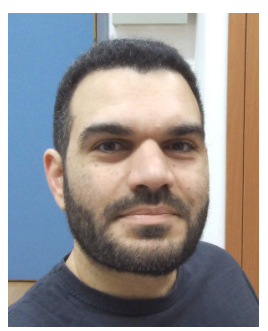

Costas Michaelides studies electrical and computer engineering with the Aristotle University of Thessaloniki (AUTh), Greece.

His research interests include the design and the performance evaluation of sensor and body area networks.

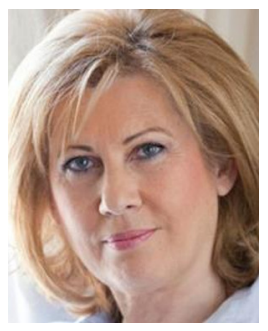

Foteini-Niovi Pavlidou (SM'00) received the Mechanical/Electrical engineering and Ph.D. degrees from the Aristotle University of Thessaloniki (AUTh), Greece.

She is currently a Full Professor with the Department of Electrical and Computer Engineering (ECE), AUTh, engaged in research and lectures in mobile networking. She has authored more than 150 papers in international journals and conferences, has been involved in various IEEE editorial activities as well as in IEEE conferences. She participated in many European and National projects. She has served as Officer for Continuing Education (CE) for IEEE Region 8 (2011- 2013), as a Coordinator of Educational Activities Subcommittee (EASC) Region 8 (2013-2015), and as a member of the IEEE Section Outreach Committee (SEOC) of EAB (20132015). 